This is the final peer-reviewed accepted manuscript of:

Semiclassical Path Integral Calculation of Nonlinear Optical Spectroscopy

Justin Provazza, Francesco Segatta, Marco Garavelli, and David F. Coker

Journal of Chemical Theory and Computation 201814 (2), 856-866

The final published version is available online at :

http://dx.doi.org/10.1021/acs.jctc.7b01063

Rights / License:

The terms and conditions for the reuse of this version of the manuscript are specified in the publishing policy. For all terms of use and more information see the publisher's website.

This item was downloaded from IRIS Università di Bologna (https://cris.unibo.it/)

When citing, please refer to the published version. 


\title{
Semiclassical Path Integral Calculation of Nonlinear Optical Spectroscopy ${ }^{\dagger}$
}

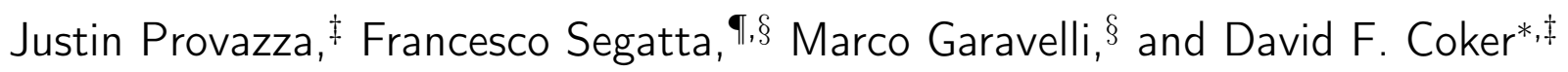 \\ $\ddagger$ Department of Chemistry, Boston University, 590 Commonwealth Avenue, Boston, \\ Massachusetts 02215, USA \\ IEuropean Center for Theoretical Studies in Nuclear Physics and Related Areas \\ (ECT*-FBK), 38123 Trento, Italy \\ $\S$ Dipartimento di Chimica Industriale "Toso Montanari", University of Bologna, Viale del \\ Risorgimento, 4, 40136 Bologna, Italy \\ E-mail: coker@bu.edu
}

\begin{abstract}
Computation of nonlinear optical response functions allows for an in-depth connection between theory and experiment. Experimentally recorded spectra provide a high density of information, but to objectively disentangle overlapping signals and to reach a detailed and reliable understanding of the system dynamics, measurements must be integrated with theoretical approaches. Here, we present a new, highly accurate and efficient trajectory based semiclassical path integral method for computing higher-order nonlinear optical response functions for non-Markovian open quantum systems. The approach is, in principle, applicable to general Hamiltonians and does not require any restrictions on the form of the intra-system or system-bath couplings. This method is

\footnotetext{
${ }^{\dagger} \mathrm{JP}$ and FS contributed equally to the development of the methods and applications that are detailed in this publication.
} 
systematically improvable and is shown to be valid in parameter regimes where perturbation theory-based methods qualitatively breakdown. As a test of the methodology presented here, we study a system-bath model for a coupled dimer for which we compare against numerically exact results and standard approximate perturbation theory based calculations. Additionally, we study a monomer with discrete vibronic states that serves as the starting point for future investigation of vibronic signatures in nonlinear electronic spectroscopy.

\section{Introduction}

Experimental nonlinear spectroscopic techniques such as time-resolved 1-dimensional pump probe (1DPP) methods and 2-dimensional electronic spectroscopy (2DES) contain enormous amounts of detailed information about relaxation processes that ensue after photo excitation. These techniques, for example, can now be applied to large arrays of chromophores such as the pigment-protein light harvesting complexes that initiate photosynthesis, ${ }^{1}$ or to new nano structured materials where processes like multiple exciton generation can potentially enhance solar cell function. ${ }^{2}$ These powerful nonlinear spectroscopies can thus provide detailed information on the ultrafast excitation energy transfer (EET) and charge transfer (CT) and separation processes in light harvesting. Beating features are ubiquitous in these signals and have highlighted the existence of long-lived vibronic coherence that may play a role in enhancing the light harvesting function of these structures. ${ }^{1}$ This has motivated reconsideration of the role that intra- and inter-molecular vibrational dynamics plays during

EET and CT processes in biological light harvesting complexes (LHCs), for example. ${ }^{1,3,4}$

While these experimental results can, in principle, provide important details about these processes, the complexity of these spectra makes a thorough understanding of the origin of particular signals a very difficult task. Overlapping signals that arise from different energetic pathways make the interpretation of experimentally recorded spectral features nontrivial. Because of this, theoretical methods that are able to reliably reproduce spectroscopic ex- 
periments are a necessity. The ability to selectively suppress individual degrees of freedom (DOFs) in computational models of these complex systems allows for the previously-hopeless assignment of many of the features of nonlinear spectra. ${ }^{5}$

Existing methods for the computation of nonlinear electronic spectra have shown great utility as tools for aiding the interpretation of experimental results. These methods such as those based on extensions to Redfield theory,${ }^{6}$ quantum master equation approaches, ${ }^{7-10}$ wave packet based techniques, ${ }^{11}$ etc. are often perturbative in nature, e.g. they assume that couplings of the sites to their environments are small compared to other terms. In addition, the more standard methods for computing the nonlinear response may make dynamical approximations about time scales of different processes (e.g. the Markovian and Secular approximations). Recent simulations that attempt to compute the parameters in the model Hamiltonians describing these types of systems from first principles ${ }^{12}$ suggest that they can fluctuate considerably as the system samples its configuration space, so using model parameters fit to averaged experimental results may not in general reliably capture the influence of these fluctuations. Further, the magnitude of the parameter fluctuations can, in principle, push the system between regimes where different perturbative approximations make sense while others become nonphysical. Such fluctuations and variations in timescales can invalidate the different perturbation theories or dynamical assumptions on which these approaches are based, limiting their usefulness as predictive methods.

To address these issues, non-perturbative methods that avoid different dynamical approximations have been developed such as the density matrix based hierarchical equations of motion (HEOM) approach ${ }^{13-16}$ or the wave function based multi-configuration time dependent Hartree (MCTDH) scheme. ${ }^{17,18}$ These numerically exact techniques have been extended to compute nonlinear optical responses in recent benchmark studies providing critical tests for more approximate methods. Implementing these exact techniques to compute the nonlinear optical response for larger more realistic models, however, can quickly become prohibitively expensive. Recently, schemes that use these types of exact methods to compute the rapidly 
decaying memory kernel in master equation approaches based on the Liouville-von Neuman equation, which can then be integrated to longer times ${ }^{19,20}$ have appeared and may offer efficient ways to compute the nonlinear optical response. A mixed quantum-classical Liouville approach to nonlinear spectroscopy has been presented and applied to compute nonlinear infrared spectroscopy. ${ }^{21-23}$ This approach employs a Wigner approximation that involved propagating classical trajectories from a quantum initial distribution to describe the vibrational dynamics underlying the nonlinear infrared response. The approach we develop here, however, takes a different, but related semiclassical tack.

We present a non-perturbative, non-Markovian semiclassical path integral method for the computation of nonlinear optical response functions in open quantum systems that explicitly treats all DOFs. ${ }^{24,25}$ This method makes no assumptions about the nature of intra-system couplings or the form of the spectral density that defines the frequency-dependent couplings between the system and its environmental DOFs, providing a way of accurately treating the coupled electronic, vibrational and vibronic dynamics at the heart of the nonlinear optical response. Moreover the method is based on propagating classical-like trajectories that include effects such as quantum transitions and the decay of an initially prepared coherent superposition of system quantum states in the presence of coupling to the environment. The independent trajectory nature of the approach makes for a highly efficient implementation for computing nonlinear responses and results in a flexible approach with good scaling to larger numbers of quantum states and explicit bath degrees of freedom.

The outline of the paper is as follows: First the general theory of nonlinear optical response functions is summarized. Next we present an approach for propagating dynamical operators based on the mapping Hamiltonian formulation for systems with discrete quantum states coupled to a bath described by continuous coordinates. This approach is based on the partial linearized density matrix (PLDM) dynamics method, ${ }^{24,25}$ only now applied for arbitrary operators. The partial linearized operator dynamics expressions for computing optical response functions are then presented and an algorithm for their efficient implementation is 
outlined. Results for 2DES for several simple model Hamiltonians involving quantum subsystems including electronic and vibrational states coupled to different baths are presented along with comparisons to exact HEOM results and results obtained from other approximate theoretical approaches. Finally some concluding observations are summarized.

\section{Theory}

\section{Optical Response Functions}

To describe the time evolution of a system in the presence of an external electromagnetic field, the field-matter Hamiltonian is partitioned as

$$
\hat{H}_{t o t}(t)=\hat{H}+\hat{H}^{\prime}(t)
$$

where $\hat{H}$ is the molecular Hamiltonian that describes the time evolution of the full system in the absence of the electromagnetic field and $\hat{H}^{\prime}(t)=-\hat{\vec{\mu}} \cdot \vec{E}(\vec{r}, t)$ represents the interaction of the system with a classical external electromagnetic field, $\vec{E}(\vec{r}, t)$. For clarity, in what follows we will omit the $\vec{r}$ dependence of the electric field as well as the vectorial nature of both the field and molecular dipole operator, $\hat{\vec{\mu}}^{1}$

Perturbation theory in the field matter interaction gives that the signal that is order $n$ in the field strength can be obtained from the $n^{t h}$-order macroscopic polarization, which reads

$$
P^{(n)}=\operatorname{Tr}\left[\hat{\mu} \hat{\rho}^{(n)}\right]
$$

where $n$ is the number of times the sample has interacted with the external electromagnetic field, $\hat{\rho}^{(n)}=\left[\hat{\mu}, \hat{\rho}^{(n-1)}\right]$ is the $n^{t h}$ order perturbed density operator and $\rho^{(0)}$ is the ground state

\footnotetext{
${ }^{1}$ The expressions given here can be generalized in a straightforward way to incorporate this relative field and molecular dipole orientational information by recognizing that each laser pulse in a typical experiment has its electric field polarized in a specific direction, perpendicular to the direction of propagation of the pulse, so the orientation dependence of the light-matter interaction for each pulse in the above expression is influenced by the dot product of the molecular transition dipole and the field polarization vector associated with the particular pulse.
} 
density matrix prior to any field interaction. In third-order experiments, the sample interacts with three laser pulses having wave vectors $\left( \pm \vec{k}_{1}, \pm \vec{k}_{2}, \pm \vec{k}_{3}\right)$. After the initial interaction, the system (prepared by the first pulse in a coherence between the ground and first excited state manifolds) is allowed to evolve for a time $t_{1}$ before the second pulse arrives. We similarly define $t_{2}$ as the time interval between the second and third pulses. During this $t_{2}$ interval the excited system interacts with its environment causing relaxation, potentially involving excitation energy transfer to other excited states and decoherence arising from entanglement of the evolving system state and its surroundings. Monitoring nonlinear spectroscopic signals as functions of $t_{2}$ can thus provide detailed information about these excited state processes. At a time delay $t_{3}$ after interacting with the third pulse, a response is emitted from the sample in a direction that is the sum of the incoming wave vectors of the different laser pulses, as depicted in Figure 1. ${ }^{6,26}$

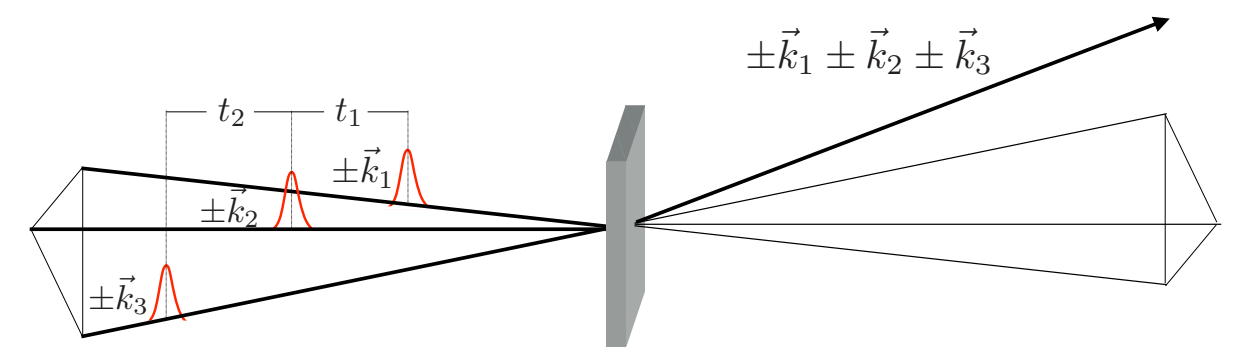

Figure 1: Pictorial representation of a third-order nonlinear spectroscopy experiment. The signal is emitted in the direction of the sum of incoming wave vectors.

The directionality of these responses allows for the independent detection of signals that correspond to different sample-field interaction schemes. The most frequently reported results for third-order experiments contain information from the rephasing and nonrephasing signals $\left(\vec{k}_{r}= \pm\left(-\vec{k}_{1}+\vec{k}_{2}+\vec{k}_{3}\right)\right.$ and $\vec{k}_{n r}= \pm\left(\vec{k}_{1}-\vec{k}_{2}+\vec{k}_{3}\right)$, respectively) although the method presented here is capable of describing all possible nonlinear responses. The signals can be further partitioned into ground state bleaching (GSB), stimulated emission (SE), and excited state absorption (ESA) contributions. ${ }^{27}$ These reflect different sequences of transitions that can occur as a consequence of the field-matter interactions. These interactions are 
conveniently summarized by double-sided Feynman diagrams and, in Figure 2, we show an example of these diagrams for the nonrephasing signal.

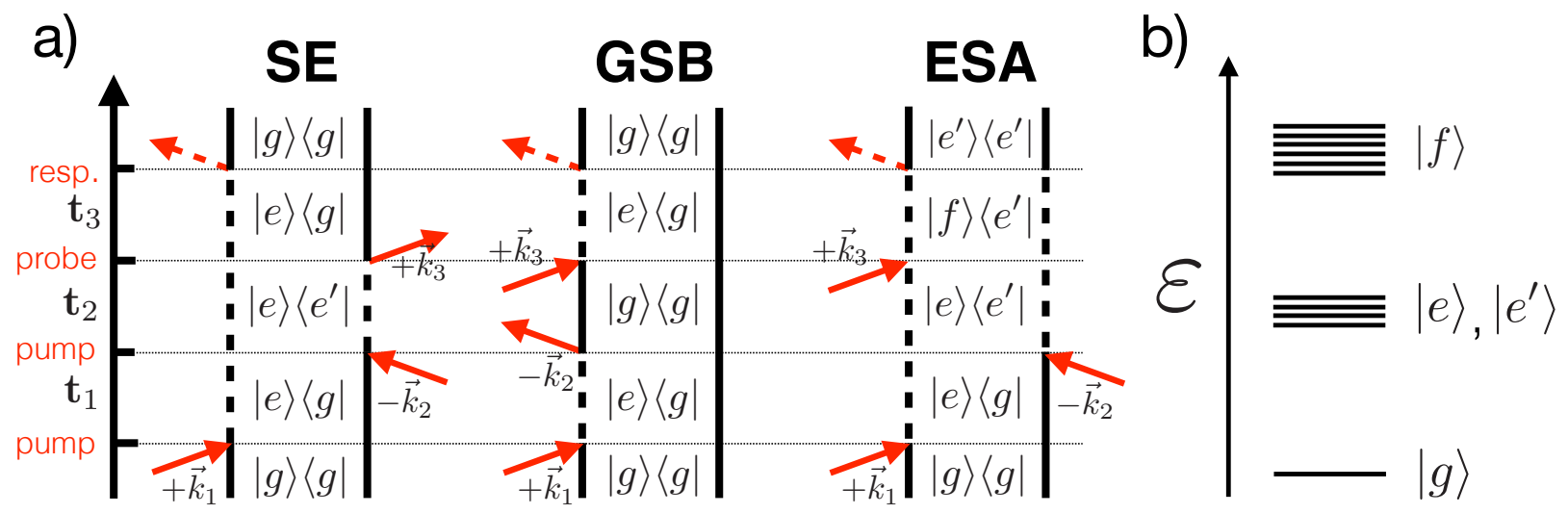

Figure 2: a) Double-sided Feynman diagrams that represent nonrephasing interaction schemes. For conciseness, only diagrams that survive the rotating wave approximation are shown. Red arrows indicate an interaction with the external field. The vertical lines represent time evolution, with dashed lines indicating time periods where the corresponding wave function can mix into a linear combination of eigenstates. Note that, in our methodology, mixing is allowed when the system is in a coherence, not just when it is in a population. b) Pictorial representation of the excitation manifolds involved in the Feynman diagrams depicted in a).

\section{Partial Linearized Operator Dynamics}

The propagation scheme we develop here to evolve the operators that appear in expressions for the nonlinear optical responses is a straightforward generalization of the partial linearized density matrix (PLDM) dynamics approach, ${ }^{24}$ and will henceforth be referred as PLDM (although it is not restricted to the propagation of density matrices ${ }^{24,25}$ ). Here we outline the principle ingredients of this approach and generalize it to evolving arbitrary operators.

The time evolution of the expectation value of a general operator $\langle\hat{O}\rangle(t)=\left\langle\Psi\left|e^{i H t} \hat{O} e^{-i H t}\right| \Psi\right\rangle$, where $H$ is a general system-bath Hamiltonian and the system and bath are prepared in some initial state $|\Psi\rangle$, can be accurately described using a semiclassical path integral formalism for the forward $\left(e^{-i H t}\right)$ and backward $\left(e^{i H t}\right)$ propagators. ${ }^{28}$ Employing the Meyer-Miller mapping model to a general system-bath Hamiltonian in the diabatic representation, the discrete 
quantum states of the system are exactly mapped onto a set of fictitious harmonic oscillator raising and lowering operators $\left(|\alpha\rangle \rightarrow \hat{a}_{\alpha}^{\dagger}=\left(\hat{x}_{\alpha}-i \hat{p}_{\alpha}\right) / \sqrt{2}\right)$ and the Hamiltonian takes the form $^{29,30}$

$$
\hat{H}=\frac{\hat{P}^{2}}{2 M}+\frac{1}{2} \sum_{\alpha} H_{\alpha \alpha}(\hat{R})\left(\hat{x}_{\alpha}^{2}+\hat{p}_{\alpha}^{2}-1\right)+\frac{1}{2} \sum_{\beta \neq \alpha} H_{\alpha \beta}(\hat{R})\left(\hat{x}_{\alpha} \hat{x}_{\beta}+\hat{p}_{\alpha} \hat{p}_{\beta}\right)
$$

where $(\hat{x}, \hat{p})$ and $(\hat{P}, \hat{R})$ represent the complete sets of system and bath phase-space operators, respectively, and we take $\hbar=1$.

To arrive at the partial linearized operator dynamics algorithm, the Herman-Kluk ${ }^{31,32}$ (or coherent state) semiclassical propagator is employed for the system DOFs. ${ }^{2}$ The bath part of the full propagator is written in phase space path integral form, and a (forward) propagator matrix element takes the form

$$
\left\langle n_{t}, R_{N}\left|e^{-i \hat{H} t}\right| n_{0}, R_{0}\right\rangle=\left(\prod_{k=1}^{N-1} \int d R_{k} \frac{d P_{k}}{2 \pi}\right) \int \frac{d P_{N}}{2 \pi} e^{i S_{0}(t)} T_{\left[n_{t}, n_{0}\right]}
$$

where $S_{0}(t)=\epsilon \sum_{k=1}^{N}\left[P_{k} \frac{\left(R_{k}-R_{k-1}\right)}{\epsilon}-\frac{P_{k}^{2}}{2 M}\right]$ is the bath kinetic action with time step $\epsilon$ and $T_{\left[n_{t}, n_{0}\right]}$ is the system transition amplitude, given by

$$
T_{\left[n_{t}, n_{0}\right]}=\int d x_{0} d p_{0} \frac{1}{2}\left(x_{n_{t}}+i p_{n_{t}}\right)\left(x_{n_{0}}-i p_{n_{0}}\right) e^{i S_{1}(t)} e^{-\frac{i}{2} \sum_{\alpha}\left(x_{\alpha_{t}} p_{\alpha_{t}}-x_{\alpha_{0}} p_{\alpha_{0}}\right)} e^{-\frac{1}{2} \sum_{\alpha}\left(x_{\alpha_{0}}^{2}+p_{\alpha_{0}}^{2}\right)}
$$

Here, $S_{1}(t)$ is the bath-dependent system action, which reads

$$
S_{1}(t)=\int_{0}^{t} d \tau\left[\sum_{\alpha} p_{\alpha} \dot{x}_{\alpha}-\tilde{H}(R, x, p)\right]
$$

where $\tilde{H}(R, x, p)=\frac{1}{2} \sum_{\alpha} H_{\alpha \alpha}(R)\left(x_{\alpha}^{2}+p_{\alpha}^{2}\right)+\frac{1}{2} \sum_{\alpha \neq \beta} H_{\alpha \beta}(R)\left(x_{\alpha} x_{\beta}+p_{\alpha} p_{\beta}\right)$.

\footnotetext{
${ }^{2}$ While results are independent of the specific choice of the coherent state width parameter, $\gamma$, choosing it as $\gamma=\frac{1}{2}$ has the useful consequence that the coherent state version of the semiclassical van Vleck determinant exactly cancels the troublesome $-\frac{1}{2} \sum_{\alpha} H_{\alpha \alpha}(R)$ term in Eq. (3). ${ }^{33}$ This mitigates the issue of the bath DOFs propagating on an inverted potential energy surface (PES) when the mapping variables sample regions of phase space in which $\hat{x}_{\alpha}^{2}+\hat{p}_{\alpha}^{2}<1$.
} 
Next, the forward and backward propagator matrix elements are combined and the bath phase space DOFs are transformed to mean and difference variables, defined as $\bar{R}=$ $\left(R+R^{\prime}\right) / 2$ and $Z=R-R^{\prime}$, respectively, with similar definitions for the corresponding momenta, $\bar{P}$ and $Y$. The approximation made in the partial linearization scheme comes through a truncation of the functional Taylor series expansion about the mean bath phase space path, $(\bar{R}(t), \bar{P}(t))$, to linear order in the difference path variables, $Z(t)$ and $Y(t)$. This approximation is based on the assumption that forward and backward bath paths will remain close to each other for short times. Moreover, we use the approximation that $\sum_{\alpha} p_{\alpha} \dot{x}_{\alpha}-\tilde{H}(\bar{R}, x, p) \approx \sum_{\alpha} p_{\alpha} \dot{x}_{\alpha}-\tilde{H}(R, x, p)=\frac{1}{2} \frac{d}{d \tau}\left(\sum_{\alpha} p_{\alpha} x_{\alpha}\right)$ to cancel the complex boundary terms in Eq. (5) (as well as those associated with the backward propagator) by using the result in Eq. (6). ${ }^{34}$ This approximation states that the classical (mean) bath drives nonadiabatic transitions in the quantum subsystem. Upon integration of this partial linearized approximate result over bath difference path variables $\left(e . g . Z_{0} \ldots Z_{N-1}\right)$, the final expression for the evolution of a matrix element of operator $\hat{O}$ is obtained as ${ }^{24}$

$$
\begin{aligned}
& \left\langle n_{t}, \bar{R}_{N}+\frac{Z_{N}}{2}|\hat{O}(t)| n_{t}^{\prime}, \bar{R}_{N}-\frac{Z_{N}}{2}\right\rangle=\sum_{n_{0}, n_{0}^{\prime}} \int d \bar{R}_{0} d x_{0} d p_{0} d x_{0}^{\prime} d p_{0}^{\prime} G_{0} G_{0}^{\prime} \\
& \quad \times \frac{1}{2}\left(x_{n_{t}}+i p_{n_{t}}\right)\left(x_{n_{0}}-i p_{n_{0}}\right) \frac{1}{2}\left(x_{n_{t}^{\prime}}^{\prime}-i p_{n_{t}^{\prime}}^{\prime}\right)\left(x_{n_{0}^{\prime}}^{\prime}+i p_{n_{0}^{\prime}}^{\prime}\right)\left(\prod_{k=1}^{N-1} \int d \bar{R}_{k} \frac{d \bar{P}_{k}}{2 \pi}\right) \int \frac{d \bar{P}_{N}}{2 \pi} \\
& \quad \times O_{W}^{n_{0} n_{0}^{\prime}}\left(\bar{R}_{0}, \bar{P}_{1}\right) e^{i \bar{P}_{N} Z_{N}} \prod_{k=1}^{N-1} \delta\left(\frac{\bar{P}_{k+1}-\bar{P}_{k}}{\epsilon}-F_{k}\right) \prod_{k=1}^{N} \delta\left(\frac{\bar{R}_{k}-\bar{R}_{k-1}}{\epsilon}-\frac{\bar{P}_{k}}{M}\right)
\end{aligned}
$$

This equation should be interpreted in the following way: initial nuclear DOFs are sampled from the quasi-probability distribution (the so-called partial Weyl symbol) $O_{W}^{n_{0} n_{0}^{\prime}}\left(\bar{R}_{0}, \bar{P}_{1}\right)=$ $\int d Z_{0}\left\langle n_{0}, \bar{R}_{0}+\frac{Z_{0}}{2}|\hat{O}| n_{0}^{\prime}, \bar{R}_{0}-\frac{Z_{0}}{2}\right\rangle e^{-i \bar{P}_{1} Z_{0}}$, labeled by initial system states $n_{0}$ and $n_{0}^{\prime}$, and mapping variables (system DOFs) are sampled from the Gaussian functions $G_{0}=\exp \left[-\frac{1}{2} \sum_{\alpha}\left(x_{\alpha 0}^{2}+p_{\alpha 0}^{2}\right)\right]$ and $G_{0}^{\prime}$. The system mapping DOFs are evaluated along classical-like trajectories (satisfy- 
ing Hamilton's equations for the Hamiltonian, $\tilde{H}$, defined under Eq. (6)) and the nuclear trajectories (as prescribed by the products of $\delta$-functions) are determined by an effective force $F_{k}=-\frac{1}{2} \nabla_{\bar{R}_{k}}\left(\tilde{H}(\bar{R}, x, p)+\tilde{H}\left(\bar{R}, x^{\prime}, p^{\prime}\right)\right)$ resulting from different forward, $(x, p)$, and backward, $\left(x^{\prime}, p^{\prime}\right)$, system DOFs. ${ }^{24}$ The trajectories propagated from such initial conditions provide a dynamical ensemble over which quantities can be averaged thus performing the following integrals $\sum_{n_{0}, n_{0}^{\prime}} \int d \bar{R}_{0} d x_{0} d p_{0} d x_{0}^{\prime} d p_{0}^{\prime}$, yielding the desired time evolved operator matrix elements. ${ }^{3}$

This framework offers a robust and systematically improvable means of computing the time evolution of quantum mechanical operators. Indeed, the measure of quantum mechanical accuracy can be controlled by a simple repartitioning of the system-bath Hamiltonian. However, the necessity of averaging the (often oscillatory) complex phase factors associated with the product of polynomial terms in the mapping variables in Eq. (7) can be problematic, resulting in considerable noise in computed averages at longer times requiring larger ensembles of trajectories to obtain desired convergence. Alternatively, treating this algorithm as a short-time propagator where the linearization approximation is valid introduces quantum interference effects in the bath and, in the limit of infinite time slices, converges to exact quantum mechanical results. ${ }^{25}$

\section{PLDM Computation of Optical Response Functions}

Theoretical analysis of the response of a system to an external electric field allows for the direct comparison of theory and experiment. The linear response function is responsible for the connection between linear polarization and the electric field, ${ }^{27}$

$$
P^{(1)}(t)=\int_{0}^{\infty} d t_{1} R^{(1)}\left(t_{1}\right) E\left(t-t_{1}\right)
$$

\footnotetext{
${ }^{3}$ It should be pointed out that, although the Herman-Kluk propagator is semiclassical, the Meyer-Miller Hamiltonian is at most quadratic in the system mapping variables. Therefore, this semiclassical propagator provides an exact quantum mechanical treatment of the system dynamics for fixed bath variables.
} 
where the first order response function, $R^{(1)}\left(t_{1}\right)=i \operatorname{Tr}\left[\hat{\mu}\left(t_{1}\right) \hat{\mu}^{\times}(0) \hat{\rho}(0)\right]$, gives the response of the system to a single interaction with the electric field. Here, $\hat{\mu}(t)=e^{+i \hat{H} t} \hat{\mu} e^{-i \hat{H} t}$ is the dipole operator in the interaction representation and $\hat{\mu}^{\times}(t) \bullet=[\hat{\mu}(t), \bullet]$ is the time dependent dipole commutation superoperator.

While the linear response of a system contains useful information about the relative energy gaps between ground and excited electronic states and the strength of coupling between electronic and nuclear DOFs, to study the excited state dynamics of the system one must go beyond linear response. For this purpose, we consider the third-order nonlinear response. Since the third-order polarization is the source of the emitted signal, the signal corresponding to any third-order nonlinear spectroscopy experiment can be extracted from the time-convolution of the nonlinear response function, $R^{(3)}\left(t_{3}, t_{2}, t_{1}\right)$, with the appropriate external time dependent electric field according to the following result: ${ }^{27}$

$$
\begin{aligned}
P^{(3)}(t) & =\int_{0}^{\infty} d t_{3} \int_{0}^{\infty} d t_{2} \int_{0}^{\infty} d t_{1} R^{(3)}\left(t_{3}, t_{2}, t_{1}\right) \\
& \times E\left(t-t_{3}\right) E\left(t-t_{3}-t_{2}\right) E\left(t-t_{3}-t_{2}-t_{1}\right)
\end{aligned}
$$

In typical third order experiments the field is defined by three time ordered light pulses that are shorter than the separations between them, but long compared to the oscillation period of the laser electric field. In the semi-impulsive limit, where the pulses are approximated by $\delta$-functions at times $\tau_{1}, \tau_{2}$ and $\tau_{3}$ multiplied by factors of the form $e^{ \pm i\left(\vec{k}_{i} \cdot \vec{r} \mp \omega_{i} t\right)}$, the integrand is only finite when $t_{3}=t-\tau_{3}, t_{2}=\tau_{3}-\tau_{2}$, and $t_{1}=\tau_{2}-\tau_{1}$ so the signal is directly obtained as the response function $R^{(3)}\left(t_{3}, t_{2}, t_{1}\right),{ }^{6}$ which can be written in terms of the multi-point dipole correlation function as:

$$
\begin{aligned}
R^{(3)}\left(t_{3}, t_{2}, t_{1}\right) & =(i)^{3}\left\langle\hat{\mu}\left(t_{3}+t_{2}+t_{1}\right) \hat{\mu}^{\times}\left(t_{2}+t_{1}\right) \hat{\mu}^{\times}\left(t_{1}\right) \hat{\mu}^{\times}(0)\right\rangle \\
& =(i)^{3} \operatorname{Tr}\left[\hat{\mu}\left(t_{3}+t_{2}+t_{1}\right) \hat{\mu}^{\times}\left(t_{2}+t_{1}\right) \hat{\mu}^{\times}\left(t_{1}\right) \hat{\mu}^{\times}(0) \hat{\rho}(0)\right]
\end{aligned}
$$


The third order signals are often presented as functions of frequencies $\Omega_{1}$ and $\Omega_{3}$ for different $t_{2}$ delay times by Fourier transformation of the third order response, $R^{(3)}\left(t_{3}, t_{2}, t_{1}\right) \rightarrow$ $\tilde{R}^{(3)}\left(\Omega_{3}, t_{2}, \Omega_{1}\right)$.

The PLDM framework has been shown to be capable of accurately reproducing quantum autocorrelation functions ${ }^{35,36}$ and we employ it here to compute the multi-point dipole autocorrelation function needed for third-order optical spectroscopy according to the following expression:

$$
\begin{aligned}
& R^{(3)}\left(t_{3}, t_{2}, t_{1}\right)=(i)^{3} \sum_{n_{t_{3}}}\left(\prod_{k=N_{2}+N_{1}+1}^{N_{3}+N_{2}+N_{1}} \int d \bar{R}_{k} \frac{d \bar{P}_{k}}{2 \pi}\right) \sum_{n_{t_{2}}, n_{t_{2}}^{\prime}} \int d \bar{R}_{N_{2}+N_{1}} \frac{d \bar{P}_{N_{2}+N_{1}}}{2 \pi} d x_{t_{2}} d p_{t_{2}} d x_{t_{2}}^{\prime} d p_{t_{2}}^{\prime} G_{t_{2}} G_{t_{2}}^{\prime} \\
& \times\left(\mu \rho^{(3)}\right)_{n_{t_{3}} n_{t_{3}}} \prod_{k=N_{2}+N_{1}+1}^{N_{3}+N_{2}+N_{1}-1} \delta\left(\frac{\bar{P}_{k+1}-\bar{P}_{k}}{\epsilon}-F_{k}\right) \prod_{k=N_{2}+N_{1}+1}^{N_{3}+N_{2}+N_{1}} \delta\left(\frac{\bar{R}_{k}-\bar{R}_{k-1}}{\epsilon}-\frac{\bar{P}_{k}}{M}\right) \delta\left(\bar{P}_{N_{2}+N_{1}+1}-\bar{P}_{N_{2}+N_{1}}\right) \\
& \times \delta\left(\frac{\bar{R}_{N_{2}+N_{1}}-\bar{R}_{N_{2}+N_{1}-1}}{\epsilon}-\frac{\bar{P}_{N_{2}+N_{1}}}{M}\right)\left(\prod_{k=N_{1}+1}^{N_{2}+N_{1}-1} \int d \bar{R}_{k} \frac{d \bar{P}_{k}}{2 \pi}\right) \sum_{n_{t_{1}}, n_{t_{1}}^{\prime}} \int d \bar{R}_{N_{1}} \frac{d \bar{P}_{N_{1}}}{2 \pi} d x_{t_{1}} d p_{t_{1}} d x_{t_{1}}^{\prime} d p_{t_{1}}^{\prime} G_{t_{1}} G_{t_{1}}^{\prime} \\
& \times\left(\mu^{\times} \rho^{(2)}\right)_{n_{t_{2}} n_{t_{2}}^{\prime}} \prod_{k=N_{1}+1}^{N_{2}+N_{1}-1} \delta\left(\frac{\bar{P}_{k+1}-\bar{P}_{k}}{\epsilon}-F_{k}\right) \prod_{k=N_{1}+1}^{N_{2}+N_{1}} \delta\left(\frac{\bar{R}_{k}-\bar{R}_{k-1}}{\epsilon}-\frac{\bar{P}_{k}}{M}\right) \delta\left(\bar{P}_{N_{1}+1}-\bar{P}_{N_{1}}\right) \\
& \times \delta\left(\frac{\bar{R}_{N_{1}}-\bar{R}_{N_{1}-1}}{\epsilon}-\frac{\bar{P}_{N_{1}}}{M}\right)\left(\prod_{k=1}^{N_{1}-1} \int d \bar{R}_{k} \frac{d \bar{P}_{k}}{2 \pi}\right) \sum_{n_{0}, n_{0}^{\prime}} \int d \bar{R}_{0} d x_{0} d p_{0} d x_{0}^{\prime} d p_{0}^{\prime} G_{t_{0}} G_{t_{0}}^{\prime} \\
& \times\left(\mu^{\times} \rho^{(1)}\right)_{n_{t_{1}} n_{t_{1}}^{\prime}} \prod_{k=1}^{N_{1}-1} \delta\left(\frac{\bar{P}_{k+1}-\bar{P}_{k}}{\epsilon}-F_{k}\right) \prod_{k=1}^{N_{1}} \delta\left(\frac{\bar{R}_{k}-\bar{R}_{k-1}}{\epsilon}-\frac{\bar{P}_{k}}{M}\right)\left(\mu^{\times} \rho^{(0)}\right)_{W}^{n_{0} n_{0}^{\prime}}\left(\bar{R}_{0}, \bar{P}_{1}\right)
\end{aligned}
$$

Equations (10) and (7) have been used to obtain this result including a trace over all DOFs, as outlined in the SI. Here $\left(\hat{\mu} \hat{\rho}^{(j)}\right)_{n_{t_{j}} n_{t_{j}}^{\prime}}$ are the matrix elements of the product of the transition dipole operator and the evolved density operator after the $j^{\text {th }}$ field interaction, i.e., from Eq.(7), these matrix elements of the $j^{\text {th }}$ order perturbed propagated density operator are computed from the time evolved mapping variables as $\hat{\rho}_{k_{t_{j}} l_{t_{j}}}^{(j)}=\frac{1}{2}\left(x_{k_{t_{j}}}+i p_{k_{t_{j}}}\right)\left(x_{n_{t_{j-1}}}-\right.$ 
$\left.i p_{n_{t_{j-1}}}\right) \frac{1}{2}\left(x_{l_{t_{j}}}^{\prime}-i p_{l_{t_{j}}}^{\prime}\right)\left(x_{n_{t_{j-1}}^{\prime}}^{\prime}+i p_{n_{t_{j-1}}^{\prime}}^{\prime}\right)$ and $G_{t_{j}}=\exp \left[-\frac{1}{2} \sum_{\alpha}\left(x_{\alpha_{t_{j}}}^{2}+p_{\alpha_{t_{j}}}^{2}\right)\right]$ (with a similar definition for $\left.G_{t_{j}}^{\prime}\right)$ is the initial distribution of mapping variables for the $(j+1)^{\text {th }}$ time interval. Finally in Eq. (11), $N_{1}, N_{2}$, and $N_{3}$ are the number of bath steps in each of the time intervals $t_{1}, t_{2}$, and $t_{3}$ respectively. ${ }^{4}$

Due to the state-collapsing nature of the dipole operator, each time it is applied it projects the evolving operator onto a different subspace of the global Hilbert space (see Figure 3). Therefore, after a dipole interaction, each matrix element can potentially give rise to a large number of new matrix elements that need to be propagated. From Eq. (11) the sums over the starting state indices at each of the interaction times have the potential to dramatically increase the number of terms that must be evolved, and each in principle requires propagating many independently sampled trajectories. This suggests that the complete treatment of realistic systems will be very computationally demanding with a brute-force implementation.

To mitigate this problem, a Monte-Carlo importance sampling procedure is employed to treat the intermediate state sums for the interactions at $t_{1}$ and $t_{2}$ by selecting the "most important" matrix element as the sole initially-occupied state for subsequent propagation. Also, the intermediate mapping variable integrals are evaluated using a steepest descent approximation. This method, termed "focusing" (the basic ingredients of which have already been presented in the literature ${ }^{37}$ ), drastically reduces the computational effort needed to compute multi-point correlation functions in the PLDM framework. ${ }^{5}$

This algorithm can be summarized for a general n-state system as follows:

1. At $\mathrm{t}=0$, the dipole commutator prepares the system in a coherence between the ground and single exciton manifold (e.g. $|e\rangle\langle g|$ ) and initial system mapping variables are sampled from Gaussian distributions as indicated in Eq.(11). Assuming the bath DOFs are unaffected by the electronic dipole operator (the Condon approximation), these variables are sampled from their equilibrium distribution on the ground electronic

\footnotetext{
${ }^{4}$ Note: The $k$ indices labeling the terms in the products in Eq.(11) apply only to the quantities immediately to the right of the product.

${ }^{5}$ When evaluating linear response functions this focusing procedure is unnecessary because there is only a single time interval.
} 


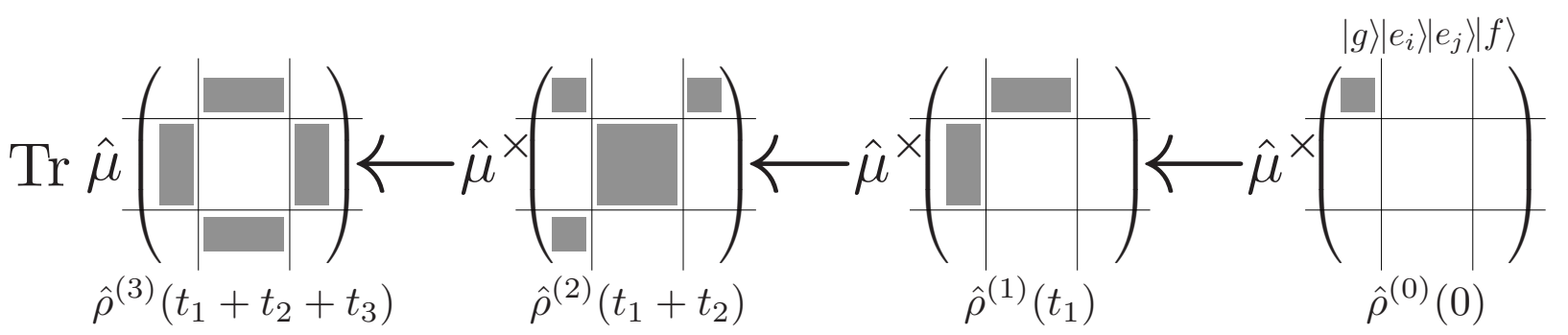

Figure 3: Pictorial representation of the effect of the subsequent dipole interactions on the evolving operator, represented as a matrix in which filled grey sectors correspond to nonzero elements of selected subspaces of the global Hilbert space. The arrows indicate time propagation.

state. An ensemble of trajectories, originating from these system and bath initial conditions is then initiated.

2. The system DOFs are propagated for a time $t_{1}$ using classical trajectories that satisfy Hamilton's equations obtained from the mapping Hamiltonian in Eq. (3). The bath DOFs are propagated simultaneously according to the effective forces, $F_{k}$ defined under Eq. (7), which depend on (both forward and backward) system mapping variables, as outlined in Eq. (11).

3. At $t=t_{1}$, the dipole commutator is applied again and the system is projected into a new set of states, while the bath DOFs remain continuous through the operation of the dipole. For each state of the projected subspace, mapping variables are re-sampled from gaussian distributions.

- If "focusing" is applied then the "most important" matrix element is selected as the new initial condition for subsequent propagation, according to the following steps:

a) The elements of the dipole operated density matrix are written, in polar coordinates, as:

$$
\left(\mu \rho^{(1)}\right)_{n_{t_{1}} n_{t_{1}}^{\prime}} \rightarrow r_{n_{t_{1}}} r_{n_{t_{1}}^{\prime}} e^{i\left(\theta_{n_{t_{1}}}-\theta_{n_{t_{1}}^{\prime}}\right)}
$$


and a normalized probability density function, $m_{n_{t_{1}} n_{t_{1}}^{\prime}}$, is constructed according to:

$$
m_{n_{t_{1}} n_{t_{1}}^{\prime}}=\frac{r_{n_{t_{1}}} r_{n_{t_{1}}^{\prime}}}{\mathcal{N}_{t_{1}}} ; \mathcal{N}_{t_{1}}=\sum_{n_{t_{1}} n_{t_{1}}^{\prime}} r_{n_{t_{1}}} r_{n_{t_{1}}^{\prime}}
$$

b) A uniform random number $\xi \in(0,1]$ is extracted, and the probability density $m_{n_{t_{1}} n_{t_{1}}}$ uniformly sampled (see the SI for details), selecting the "most important" matrix element $\left(n_{t_{1}}^{o}, n_{t_{1}}^{\prime o}\right)$ as the new (occupied) initial system density matrix element for the next time interval propagation

c) A trajectory dependent weight $\Omega_{t_{1}}=\mathcal{N}_{t_{1}} e^{i\left(\theta_{n_{t_{1}}^{o}}-\theta_{\left.n_{t_{1}}^{\prime o}\right)}\right.}$ is saved.

d) The "steepest descent" approximation is used to evaluate the integrals over the mapping variables resulting in the following:

$$
\left(n_{t_{1}}, n_{t_{1}}^{\prime}\right)=\left(\delta_{n_{t_{1}}, n_{t_{1}}^{o}}, \delta_{n_{t_{1}}^{\prime}, n_{t_{1}}^{\prime o}}\right)
$$

4. For each given $t_{1}$, steps $2-3$ are repeated for a desired number of $t_{2}$ values (i.e. replacing $t_{1}$ by $t_{2}$ in these steps).

5. For each combination of $t_{1}, t_{2}$ the system and bath are propagated for a time delay $t_{3}$, after which the expectation value of the third-order polarization is computed by averaging over all trajectories.

- If "focusing" is used, the appropriate Monte-Carlo weights are accumulated and applied when computing the contribution of each trajectory to the final response. 


\section{Results}

Although this formalism is applicable to any form of nonlinear spectroscopy, for "proof-ofconcept" purposes, here we present 2DES results for several benchmark problems to explore the reliability of the approach in various parameter regimes. The focusing approximation has been employed for all of the models studied. As mentioned above, these calculations were performed in the impulsive limit and they ignore the vectorial nature of the radiationmatter interactions, however, general pulse shapes and relative orientations of laser field polarization and molecular dipoles can be readily incorporated. It should be noted that our calculation based on Eq. (11) includes all contributions to the third order response. The rotating wave approximation was applied for the analysis of the computed response functions, and the various contributions (GSB, SE, ESA) disentangled. We note however, that this approximation can also easily be relaxed, and non-resonant contributions included. Additionally, for the results presented here, the integrals over initial mapping variables were evaluated using the "steepest descent" approximation. It was found that fully sampling the initial mapping distribution did not significantly affect the results.

The Frenkel-Exciton system-bath Hamiltonian has found great utility as a model to represent the energetics of molecular aggregates. As such, we have employed this model to describe the time evolution of the full system in the absence of an electromagnetic field. Assuming that the system and bath are initially in a product state, $\hat{\rho}^{(0)}=\hat{\rho}_{s}^{(0)} \hat{\rho}_{b}^{(0)}$, with the bath in thermal equilibrium, the initial harmonic bath DOFs are sampled from the Wigner transform of the bath Boltzmann operator.

$$
\left(\hat{\rho}_{b}^{(0)}\right)_{W} \propto \prod_{k=1}^{N_{o s c}} \exp \left[-\frac{2 \tanh \left(\beta \omega_{k} / 2\right)}{\omega_{k}}\left(\frac{P_{k}^{2}}{2}+\frac{\omega_{k}^{2} Q_{k}^{2}}{2}\right)\right]
$$

The system is assumed to be initially in its ground state. 


\section{Coupled Dimer}

In our first test of the methodology, the 2DES of a coupled dimer was computed. In this model we assume a pair of coupled sites, each of which has two levels, i.e. each site has a significant gap between its first, and all the other excited states. The manifold of coupled dimer states probed during a third-order nonlinear spectroscopy experiment is completely described by the site basis states, $|i j\rangle$, so that a general time dependent state has the form:

$$
|\psi(t)\rangle=\sum_{i, j=0}^{1} C_{i j}(t)|i j\rangle
$$

where $i$ and $j$ represent the electronic state of each site. The Hamiltonian in this representation becomes

$$
\begin{aligned}
\hat{H}=\sum_{i, j=0}^{1} \epsilon_{i j}|i j\rangle\langle i j| & +\sum_{i j \in 01,10} \Delta_{i j, j i}|i j\rangle\langle j i| \\
& +\sum_{i j \in 01,10,11} \sum_{m=1}^{M} c_{i j}^{m} \hat{R}_{m}|i j\rangle\langle i j|+\sum_{m=1}^{M} \frac{1}{2}\left(\hat{P}_{m}^{2}+\omega_{m}^{2} \hat{R}_{m}^{2}\right) \hat{\mathbb{1}}
\end{aligned}
$$

where $\mathbf{c}$ is the strength of the linear system-bath coupling, with $\mathbf{c}_{11}=\mathbf{c}_{10}+\mathbf{c}_{01}$, and $\epsilon_{11}=$ $\epsilon_{10}+\epsilon_{01}$. The spectral density that determines the bath mode frequencies and the frequency dependent system-bath coupling constants is of the Ohmic-Lorentz model form, $J(\omega)=$ $2 \lambda \frac{\omega / \omega_{c}}{\left(1+\left(\omega / \omega_{c}\right)^{2}\right)}=\frac{\pi}{2} \sum_{m} \frac{c_{m}^{2}}{\omega_{m}} \delta\left(\omega-\omega_{m}\right)$. In the calculations carried out here, each site was coupled to its own independent identical set of $M=100$ bath oscillators sampled from this distribution. ${ }^{38}$

As a test of the validity of the method presented here, two different coupled dimer models were considered.

First, to demonstrate the applicability of the PLDM 2DES approach in parameter regimes where perturbation theory-based methods can become inaccurate, we considered a dimer 


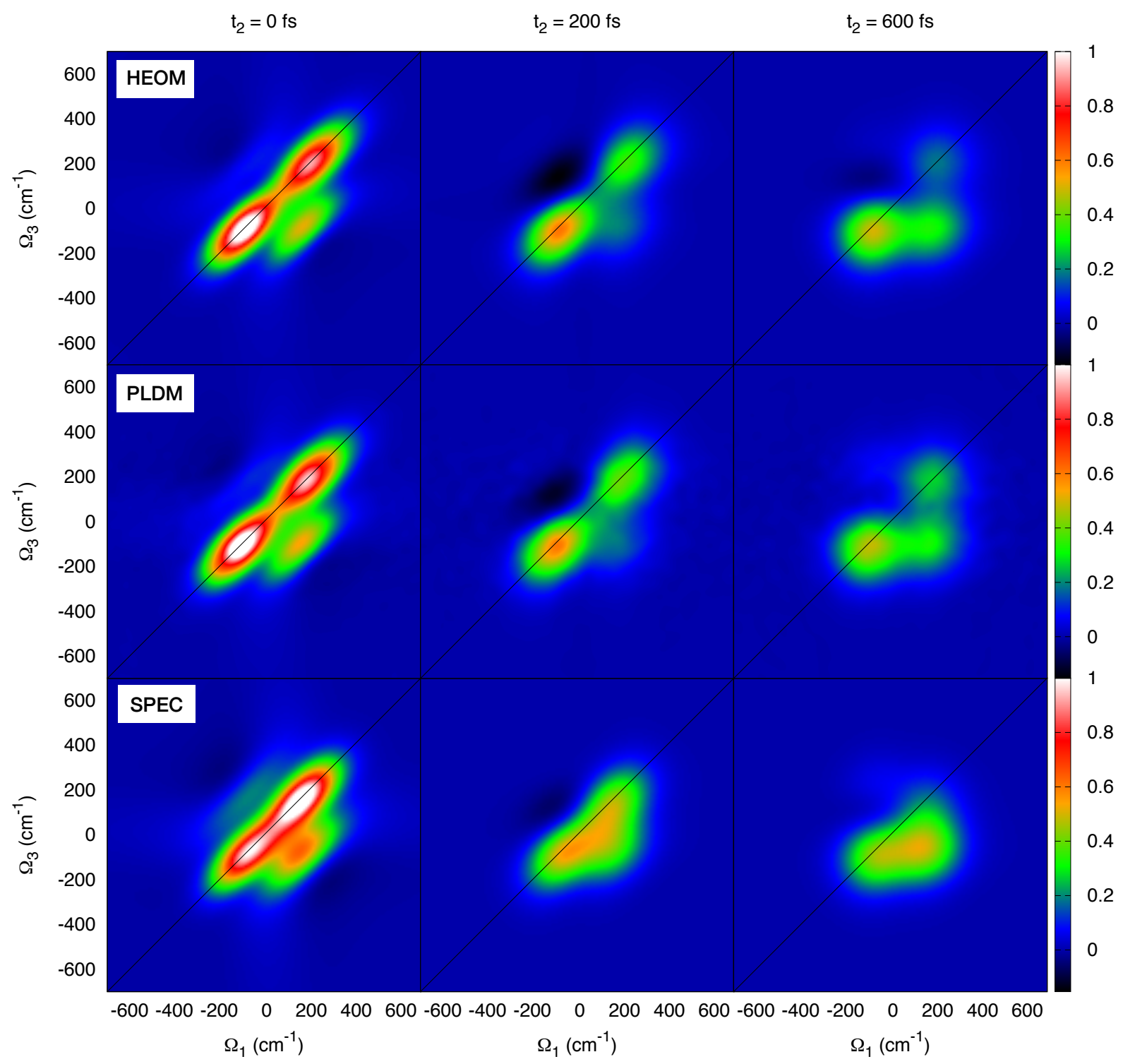

Figure 4: Comparison of HEOM, PLDM, and SPECTRON 2DES at different $t_{2}$ times for a coupled dimer at $300 \mathrm{~K}$ with $\epsilon_{10}-\epsilon_{01}=100 \mathrm{~cm}^{-1}, \Delta_{01,10}=100 \mathrm{~cm}^{-1}$, and $\mu_{00,01} / \mu_{00,10}=$ -0.2 . The bath has a cutoff frequency of $\omega_{c}=18 \mathrm{~cm}^{-1}$ and a reorganization energy of $\lambda=50 \mathrm{~cm}^{-1}$. The PLDM calculation presented here is averaged over only 60,000 trajectories initialized from each element of $\left(\mu^{\times} \rho^{(0)}\right)$ with nonzero amplitude. Note: The color range has been scaled to highlight the midrange features as detailed in the SI

system coupled to a bath with a slow relaxation time $\left(\omega_{c}^{-1}=300 \mathrm{fs}\right)$. In the limit of slow bath relaxation, the Markovian approximation can become unphysical. In Figure 4 the 2DES results computed with the PLDM approach are seen to reproduce those obtained from numerically exact hierarchical equations of motion (HEOM) calculations with surpris- 
ing accuracy, even when the results are averaged over a limited number of trajectories. The "SPEC" results reported in Figure 4 were computed using the SPECTRON software package ${ }^{6}$ employing line-shape functions obtained from the spectral density to account for all dephasing mechanisms in the absence of transport, and then using secular Redfield theory to describe transport. It is well known that Redfield theory can provide inaccurate transport dynamics in certain model parameter regimes, and we also notice that the use of the secular approximation not only decouples populations from coherences, but also prohibits the mix-

ing between coherences. This means that a density matrix element initiated in $|e\rangle\langle g|$ will evolve according to it's own equation of motion, without coupling with any other $\left|e^{\prime}\right\rangle\langle g|$ term. These approximations lead to qualitatively inaccurate results, even for $t_{2}=0 \mathrm{fs}$, where this approach predicts inverted relative intensities of features corresponding to the two exciton states. In recent work it was demonstrated that this coupled dimer model also exhibits the complete breakdown of the second-order time-convolutionless master equation for this range of parameters. ${ }^{10}$

Results for the second coupled dimer model considered are presented in Figure 5. This model displays a coherent energy transfer mechanism, which is revealed by oscillations of the energy transfer-related cross-peak. The majority of the increase in positivity of the energy transfer related cross-peak (below the diagonal) can be attributed to the quenching of negative ESA signal of the higher exciton state, concurrent with the energy migration process. Independent 2DES corresponding to isolated ESA or GSB and SE signals are presented in the Supporting Information.

\section{Vibronic monomer}

In many systems-of-interest, there can be higher frequency nuclear vibrational modes that are strongly coupled to the electronic transitions. ${ }^{12}$ In this case, the nuclear vibration becomes inherently quantum mechanical, and cannot be accurately treated using the approximate dynamical model that may still be reliable for other more weakly coupled bath modes. In 
a)

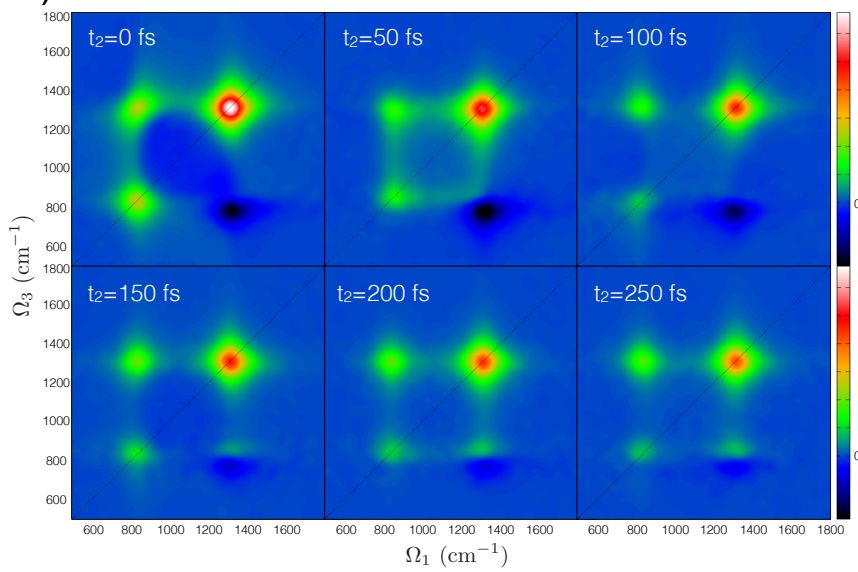

b)

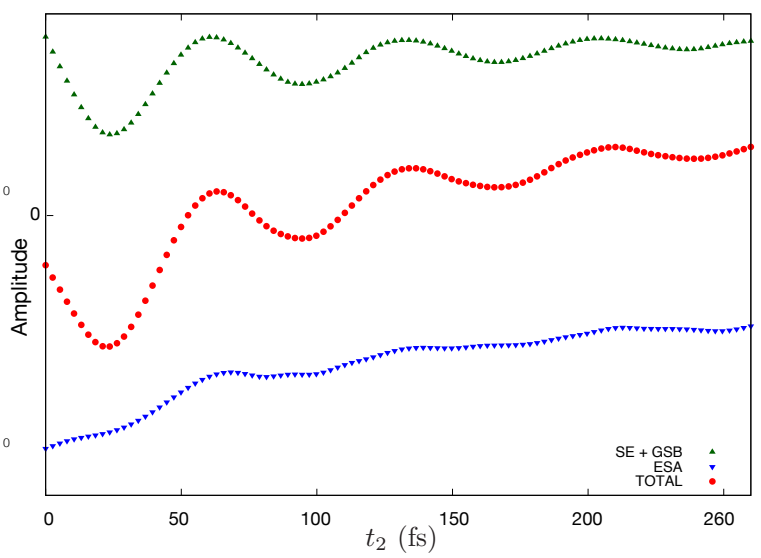

Figure 5: 2DES for a coupled dimer at $300 \mathrm{~K}$ with $\epsilon_{10}-\epsilon_{01}=200 \mathrm{~cm}^{-1}, \Delta_{01,10}=200 \mathrm{~cm}^{-1}$, and $\mu_{00,01} / \mu_{00,10}=2.4$. The spectral density parameters employed were $\lambda=50 \mathrm{~cm}^{-1}$, $\omega_{c}=200 \mathrm{~cm}^{-1}$. Panel a) shows the spectra for varying $t_{2}$ waiting time. Panel b) shows the amplitude of the energy transfer-related cross-peak at $\left(\Omega_{1}, \Omega_{3}\right) \approx(1325,875) \mathrm{cm}^{-1}$. The amplitude evolves with coherent oscillations resonant with the energy difference between exciton states.

order to track the dynamics of particular vibronic states, a repartitioning of the system-bath Hamiltonian is required. For illustrative purposes, we consider a simple vibronic monomer including one discrete harmonic vibrational mode and a single electronically excited state interacting with a continuum of $M_{c}$ more weakly coupled bath modes described by a Hamiltonian of the following form:

$\hat{H}=\sum_{n_{u}=0}^{\infty} \epsilon_{g}^{\left(n_{u}\right)}\left|g_{n_{u}}\right\rangle\left\langle g_{n_{u}}\left|+\sum_{n_{s}=0}^{\infty} \epsilon_{e}^{\left(n_{s}\right)}\right| e_{n_{s}}\right\rangle\left\langle e_{n_{s}}\left|+\sum_{n_{s}=0}^{\infty} \sum_{m=1}^{M_{c}} c_{m} \hat{R}_{m}\right| e_{n_{s}}\right\rangle\left\langle e_{n_{s}}\right|+\sum_{m=1}^{M_{c}} \frac{1}{2}\left(\hat{P}_{m}^{2}+\omega_{m}^{2} \hat{R}_{m}^{2}\right) \hat{\mathbb{1}}$

where $\left|g_{n_{u}}\right\rangle$ is the electronic ground state dressed with the state of the discrete vibrational mode with frequency $\Omega_{d}$ and in energy level $n_{u}$ centered in coordinate space about the ground electronic state's unshifted (" $u$ ") equilibrium position, $\left|e_{n_{s}}\right\rangle$ is the electronically excited state dressed with the discrete vibrational mode in energy level $n_{s}$ centered in co- 
ordinate space about the excited electronic state's shifted (" $s$ ") equilibrium position, and $\epsilon_{g}^{\left(n_{u}\right)}=\epsilon_{g}+\left(n_{u}+\frac{1}{2}\right) \Omega_{d}$ are the energies of the electronic ground state manifold. In a similar fashion, $\epsilon_{e}^{\left(n_{s}\right)}=\epsilon_{e}-\Lambda_{d}+\left(n_{s}+\frac{1}{2}\right) \Omega_{d}$ is the excitation energy at the Franck-Condon point minus the reorganization energy, $\Lambda_{d}=\frac{c_{d}^{2}}{2 \Omega_{d}^{2}}$, dressed with the discrete mode (see Figure 6).
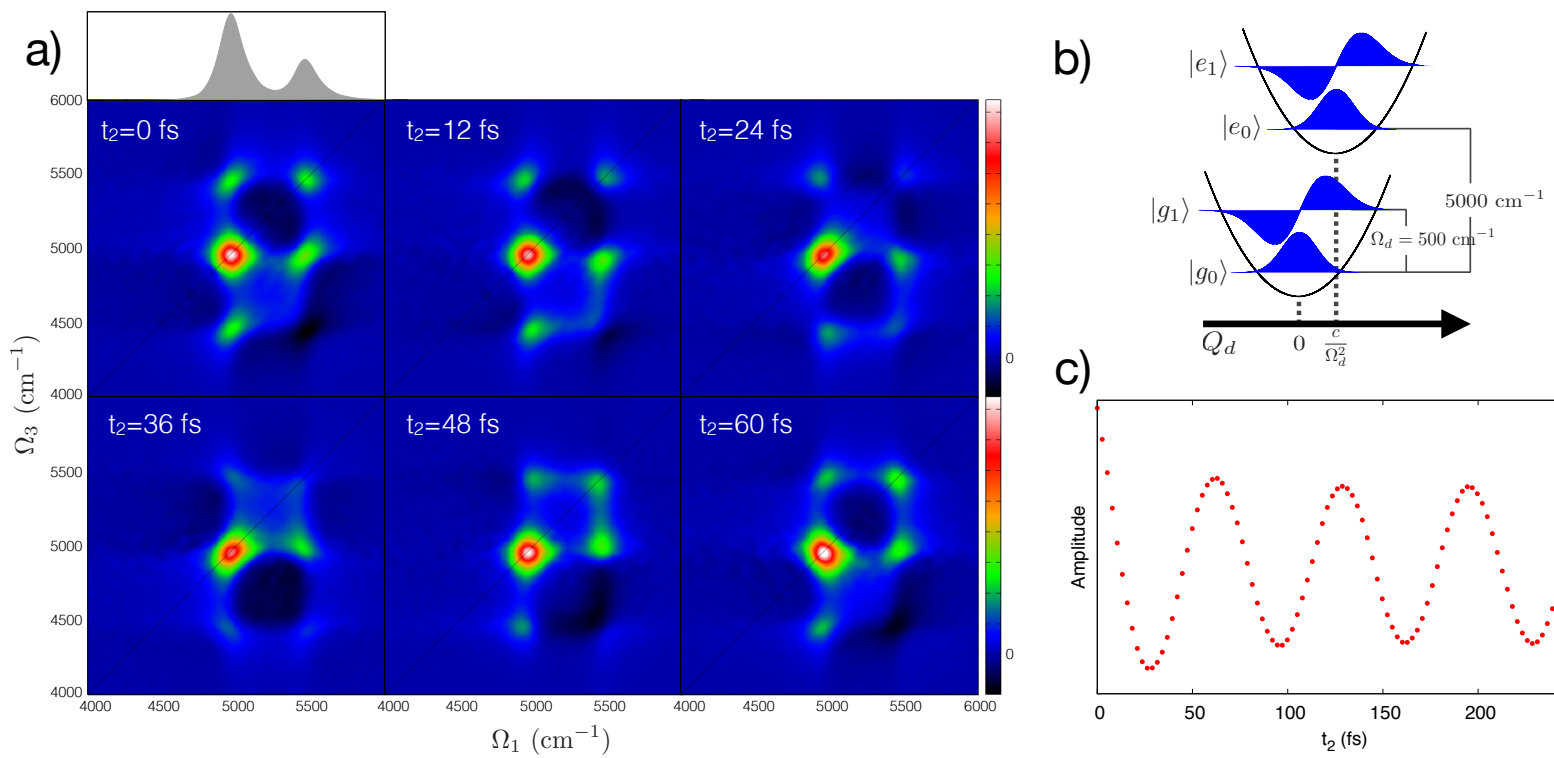

c)

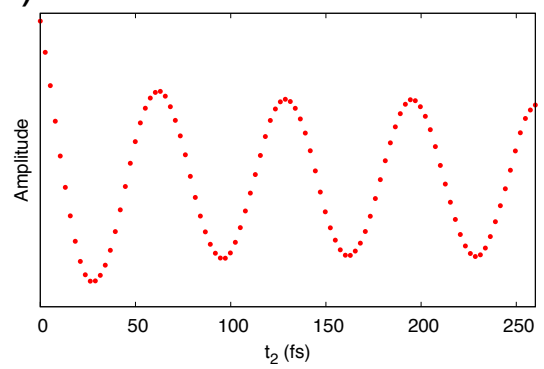

Figure 6: Summary of Vibronic Monomer study at $300 \mathrm{~K}$. The electronic system-bath coupling model spectral density is identical to that used in the coupled dimer study reported in Figure 5. The additional discrete mode in the vibronic monomer model studied here has a frequency of $\Omega_{d}=500 \mathrm{~cm}^{-1}$ and Huang-Rhys factor $S=0.45$. The $\left|g_{n}\right\rangle$ to $\left|e_{n}\right\rangle$ energy gap is $5000 \mathrm{~cm}^{-1}$. In this study the initial system density operator is a population on the total ground state $\left|g_{0}\right\rangle$. Panel a) shows the linear absorption spectrum as well as the corresponding 2DES spectra for various values of $t_{2}$. Panel b) presents a pictorial representation of the model system Hamiltonian used in this study. Panel c) shows the evolution of the off-diagonal peak located at $\left(\Omega_{1}, \Omega_{3}\right)=(5000,5500) \mathrm{cm}^{-1}$ as a function of the $t_{2}$ delay time.

In the vibronic basis, elements of the electronic dipole matrix are modulated by the overlap of shifted vibrational wavefunctions. The magnitude of the overlap is dictated by Huang-Rhys factor, $S=\Lambda_{d} / \Omega_{d}$, which indicates the relative shift between ground and excited state PESs. 


$$
\begin{aligned}
\left\langle e_{n_{s}}|\hat{\mu}| g_{m_{u}}\right\rangle & =\mu_{e g}\left\langle n_{s} \mid m_{u}\right\rangle \\
& =\mu_{e g} \int d x\left\langle n \mid x-c / \Omega_{d}^{2}\right\rangle\langle x \mid m\rangle
\end{aligned}
$$

In the calculation presented here, only vibrational energy levels 0 and 1 of the strongly coupled discrete mode were included due to the rapid fall-off of vibrational overlap for transitions between $\left|g_{0}\right\rangle \rightarrow\left|e_{n_{s}}\right\rangle$ for large Huang-Rhys factors. ${ }^{39}$

Figure 6 presents 2DES results for the vibronic system at various values of $t_{2}$. The evolution of the diagonal peaks and cross-peaks is modulated with a period of $66.7 f s$, corresponding to the $500 \mathrm{~cm}^{-1}$ frequency of the discrete mode. These signal modulations can be easily misinterpreted in experiments as a signature of electronic coherence between different sites. For example, in Figure 6 it is shown that off-diagonal features associated with pure vibrational coherence can exhibit oscillatory modulation reminiscent of peaks associated with electronic coherence. The accuracy of the dynamics method presented in this work allows us to reproduce, and correctly interpret all kinds of signal oscillations. Indeed we want to apply this machinery to realistic coupled systems in which vibronic signatures are present in order to test recent experimental results that suggest the influence of vibronic coherence on long-lived coherent energy transfer. ${ }^{4}$

Finally, we also note that the possibility of explicitly including vibronic states allows for a proper description of realistic pulse shapes: experimental pulses are in fact bandwidth limited, i.e. able to excite just some of the many possible available vibronic states. Accounting for this band-width effect using post-processing is not as accurate as actually including only the relevant set of vibronic states. This aspect, which has not been developed in the present work, will be the subject of a future study. 


\section{Conclusions}

We have presented a semiclassical path integral method for computing nonlinear optical response functions in non-Markovian open quantum systems. It combines a detailed description of the non-equilibrium dynamics responsible for energy transfer after photo-excitation in molecular aggregates (through the PLDM methodology) with response function theory of field-matter interactions, allowing for the direct connection between theory and experiment.

We have demonstrated that the method presented here is capable of producing accurate results in parameter regimes where perturbation theory-based methods breakdown significantly. ${ }^{10}$ If perturbation theory-based methodologies remain the only feasible way of treating system with a very large number of interacting chromophores, while paying the price of using approximations which limit their validity to certain parameters regimes, the methodology introduced here avoids this drawback by making no assumption about the form or strength of intra-system or system-bath couplings, even if it is generally more expensive. This method is not restricted to harmonic baths, although its accuracy in computing nonlinear spectra for anharmonic systems has yet to be studied. We also note that our approach is trajectory based and, as such, scales linearly in a parallel computation environment.

Our semiclassical path integral method offers an exciting starting point from which to explore the development of accurate, yet computationally economical, methods for computing nonlinear electronic spectroscopy. Future work will apply the present methodology to study e.g. systems of coupled vibronic sites (to clarify the influence of coherent phenomena in energy transfer) and to describe the influence of finite bandwidth pulse shapes. We are also working on the further development of the PLDM 2DES approach to decrease the computational cost and extend its applicability to larger systems, for example, by employing more efficient representations of the environmental variables. ${ }^{40-42}$ 


\section{Acknowledgement}

DFC and JP acknowledge support for this research from the National Science Foundation under grant number CHE-1665367. The authors thank Jonathan Fetherolf and Prof. Timothy Berkelbach for providing the HEOM 2D maps. MG and FS acknowledge Fondazione Bruno Kessler (FBK) and the Marco Polo program (University of Bologna) for the financial support. MG and FS thank Dr. Simone Taioli and Prof. Maurizio Dapor for the ongoing discussions and collaboration. MG acknowledges support by the European Research Council Advanced Grant STRATUS (ERC-2011-AdG No.291198) and of the French Agence National de la Recherche (FEMTO-2DNA, ANR-15-CE29-0010). We acknowledge the computational resources provided by Boston University's Office of Research Computing, Information Systems and Technology.

\section{Supporting Information Available}

The Supporting Information is available free of charge on the ACS Publications website at DOI:

It contains: some additional remarks about third order response functions, a description of the focusing Monte Carlo importance sampling procedure, details of how the color range is set for Figure 4, and animated GIF files showing the time evolution of the 2DES signals for all model systems considered. This material is available free of charge via the Internet at http://pubs.acs.org/.

\section{References}

(1) Scholes, G. D.; Fleming, G. R.; Chen, L. X.; Aspuru-guzik, A.; Buchleitner, A.; Coker, D. F.; Engel, G. S.; Grondelle, R. V.; Ishizaki, A.; Jonas, D. M.; Lundeen, J. S.; 
Mccusker, J. K. Using coherence to enhance function in chemical and biophysical systems. Nature Publishing Group 2017, 543, 647-656.

(2) Bakulin, A. A.; Morgan, S. E.; Kehoe, T. B.; Wilson, M. W. B.; Chin, A. W.; Zigmantas, D.; Egorova, D.; Rao, A. Real-time observation of multiexcitonic states in ultrafast singlet fission using coherent 2D electronic spectroscopy. Nat. Chem. 2015, 8, 16-23.

(3) Chenu, A.; Scholes, G. D. Coherence in Energy Transfer and Photosynthesis. Annu. Rev. Phys. Chem. 2015, 66, 69-96.

(4) Dean, J. C.; Mirkovic, T.; Toa, Z. S.; Oblinsky, D. G.; Scholes, G. D. Vibronic Enhancement of Algae Light Harvesting. Chem 2016, 1, 858-872.

(5) Segatta, F.; Cupellini, L.; Jurinovich, S.; Mukamel, S.; Dapor, M.; Taioli, S.; Garavelli, M.; Mennucci, B. A Quantum Chemical Interpretation of Two-Dimensional Electronic Spectroscopy of Light-Harvesting Complexes. J. Am. Chem. Soc. 2017, 139, $7558-7567$.

(6) Abramavicius, D.; Palmieri, B.; Voronine, D. V.; Sanda, F.; Mukamel, S. Coherent Multidimensional Optical Spectroscopy of Excitons in Molecular Aggregates; Quasiparticle versus Supermolecule Perspectives. Chem. Rev. 2009, 109, 2350-2408.

(7) Breuer, H.-P.; Petruccione, F. The Theory of Open Quantum Systems; Oxford University Press, New York, 2002.

(8) Shibata, F.; Takahashi, Y.; Hashitsume, N. A generalized stochastic liouville equation. Non-Markovian versus memoryless master equations. J. Stat. Phys 1977, 17, 171-187.

(9) Chaturvedi, S.; Shibata, F. Time-convolutionless projection operator formalism for elimination of fast variables. Applications to Brownian motion. Zeitschrift für Physik B Condensed Matter and Quanta 1979, 35, 297-308. 
(10) Fetherolf, J. H.; Berkelbach, T. C. Linear and nonlinear spectroscopy from quantum master equations. arXiv:1709.04075 2017,

(11) Cina, J. A.; Kovac, P. A.; Jumper, C. C.; Dean, J. C.; Scholes, G. D. Ultrafast transient absorption revisited: Phase-flips, spectral fingers, and other dynamical features. $J$. Chem. Phys. 2016, 144, 175102.

(12) Lee, M. K.; Bravaya, K. B.; Coker, D. F. First-Principles Models for Biological LightHarvesting: Phycobiliprotein Complexes from Cryptophyte Algae. J. Am. Chem. Soc. 2017, 139, 7803-7814.

(13) Tanimura, Y.; Kubo, R. Time Evolution of a Quantum System in Contact with a Nearly Gaussian-Markoffian Noise Bath. J. Phys. Soc. JPN 1989, 58, 101-114.

(14) Ishizaki, A.; Fleming, G. R. Theoretical Examination of Quantum Coherence in Photosynthetic System at Physiological Temperature. P. Natl. Acad. Sci. USA 2009, 106, $17255-17260$.

(15) Kreisbeck, C.; Kramer, T.; Rodríguez, M.; Hein, B. High-Performance Solution of Hierarchical Equations of Motion for Studying Energy Transfer in Light-Harvesting Complexes. J. Chem. Theory Comput. 2011, 7, 2166.

(16) Kreisbeck, C.; Kramer, T.; Aspuru-Guzik, A. Disentangling Electronic and Vibronic Coherences in Two-Dimensional Echo Spectra. J. Phys. Chem. B 2013, 117, 93809385 .

(17) Meyer, H.-D. Studying molecular quantum dynamics with the multiconfiguration timedependent Hartree method. WIREs Comput. Mol. Sci. 2011, 2, 351-374.

(18) Schulze, J.; Shibl, M.; Al-Marri, M.; Kühn, O. The effect of site-specific spectral densities on the high-dimensional exciton-vibrational dynamics in the FMO complex. Chem. Phys. 2017, 497, 10-16. 
(19) Makri, N. Iterative blip-summed path integral for quantum dynamics in strongly dissipative environments. J. Chem. Phys. 2017, 146, 134101.

(20) Kananenka, A. A.; Hsieh, C.-Y.; Cao, J.; Geva, E. Accurate Long-Time Mixed Quantum-Classical Liouville Dynamics via the Transfer Tensor Method. J. Phys. Chem. Lett. 2016, 7, 4809-4814.

(21) Shi, Q.; Geva, E. A comparison between different semiclassical approximations for optical response functions in nonpolar liquid solution. II. The signature of excited state dynamics on two-dimensional spectra. J. Chem. Phys. 2008, 129, 124505.

(22) Hanna, G.; Geva, E. Multidimensional spectra via the mixed quantum-classical liouville method: Signatures of nonequilibrium dynamics. J. Phys. Chem. B 2009, 113, 92789288.

(23) Mcrobbie, P. L.; Geva, E. A Benchmark Study of Different Methods for Calculating One-And Two-Dimensional Optical Spectra. J. Phys. Chem. A 2009, 113, 10425-10434.

(24) Huo, P.; Coker, D. F. Communication: Partial linearized density matrix dynamics for dissipative, non- adiabatic quantum evolution. J. Chem. Phys. 2011, 135, 201101.

(25) Huo, P.; Coker, D. F. Consistent schemes for non-adiabatic dynamics derived from partial linearized density matrix propagation. J. Chem. Phys. 2012, 137, 22A535.

(26) Hamm, P.; Zanni, M. Concepts and Methods of 2D Infrared Spectroscopy; Cambridge University Press, New York, 2011.

(27) Mukamel, S. Principles of nonlinear optical spectroscopy; Oxford University Press, New York, 1995.

(28) Bonella, S.; Coker, D. F. A semiclassical limit for the mapping Hamiltonian approach to electronically nonadiabatic dynamics. J. Chem. Phys. 2001, 114, 7778-7789. 
(29) Meyer, H.-D.; Miller, W. H. A classical analog for electronic degrees of freedom in nonadiabatic collision processes. J. Chem. Phys. 1979, 70, 3214-3223.

(30) Stock, G.; Thoss, M. Semiclassical Description of Nonadiabatic Quantum Dynamics. Phys. Rev. Lett. 1997, 78, 578-581.

(31) Herman, M. F.; Kluk, E. A semiclasical justification for the use of non-spreading wavepackets in dynamics calculations. Chem. Phys. 1984, 91, 27-34.

(32) Kay, K. G. The Herman-Kluk approximation: Derivation and semiclassical corrections. Chem. Phys. 2006, 322, 3-12.

(33) Bonella, S.; Coker, D. F. LAND-map, a linearized approach to nonadiabatic dynamics using the mapping formalism. J. Chem. Phys. 2005, 122, 194102.

(34) Lee, M. K.; Huo, P.; Coker, D. F. Semi-classical path integral dynamics: Photosynthetic energy transfer with realistic environment interactions. Annu. Rev. Phys. Chem. 2016, 67, 639-668.

(35) Bonella, S.; Montemayor, D.; Coker, D. F. Linearized path integral approach for calculating nonadiabatic time correlation functions. P. Natl. Acad. Sci. USA 2005, 102, $6715-6719$.

(36) Huo, P.; Miller, T. F.; Coker, D. F. Communication: Predictive partial linearized path integral simulation of condensed phase electron transfer dynamics. J. Chem. Phys. 2013, 139, 151103.

(37) Dunkel, E.; Bonella, S.; Coker, D. F. Iterative linearized approach to nonadiabatic dynamics. J. Chem. Phys. 2008, 129, 114106.

(38) Wang, H.; Song, X.; Chandler, D.; Miller, W. H. Semiclassical study of electronically nonadiabatic dynamics in the condensed-phase: Spin-boson problem with Debye spectral density. J. Chem. Phys. 1999, 110, 4828-4840. 
(39) Basinskaite, E.; Butkus, V.; Abramavicius, D.; Valkunas, L. Vibronic Models for Nonlinear Spectroscopy Simulations. Photosynth. Res. 2014, 121, 95-106.

(40) Garg, A.; Onuchic, J. N.; Ambegaokar, V. Effect of friction on electron transfer in biomolecules. J. Chem. Phys. 1985, 83, 4491.

(41) Hughes, K. H.; Christ, C. D.; Burghardt, I. Effective-mode representation of nonMarkovian dynamics: A hierarchical approximation of the spectral density. I. Application to single surface dynamics. J. Chem. Phys. 2009, 131, 124109.

(42) Hughes, K. H.; Christ, C. D.; Burghardt, I. Effective-mode representation of nonMarkovian dynamics: A hierarchical approximation of the spectral density. II. Application to Nonadiabatic dynamics. J. Chem. Phys. 2009, 131, 124108. 
Graphical TOC Entry

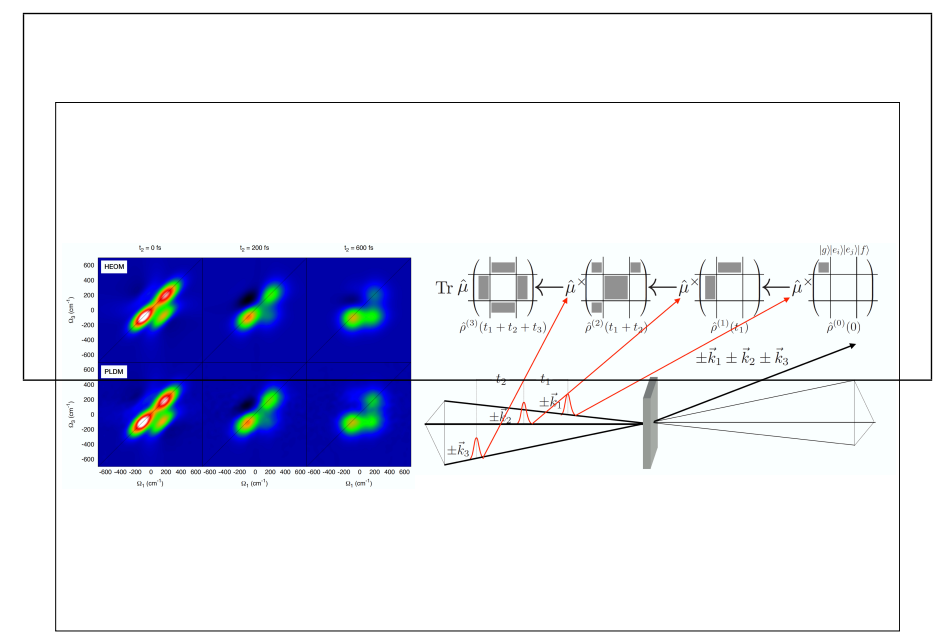

